\title{
A clustering approach to identify severe bronchiolitis profiles in children
}

\author{
Orianne Dumas, 1,2,3,4 Jonathan M Mansbach, ${ }^{2,5}$ Tuomas Jartti, ${ }^{6}$ Kohei Hasegawa, ${ }^{1,2}$ \\ Ashley F Sullivan, ${ }^{1}$ Pedro A Piedra, ${ }^{7}$ Carlos A Camargo $\mathrm{Jr}^{1,2}$
}

\begin{abstract}
- Additional material is
published online only. To view please visit the journal online (http://dx.doi.org/10.1136/ thoraxjn-2016-208535)
\end{abstract}

${ }^{1}$ Department of Emergency Medicine, Massachusetts General Hospital, Boston, Massachusetts, USA ${ }^{2}$ Harvard Medical School, Boston, Massachusetts, USA ${ }^{3}$ Inserm, VIMA, Aging and Chronic Diseases.

Epidemiological and Public Health Approaches, U1168, Villejuif, France

${ }^{4}$ UMR-S 1168, University Versailles St-Quentin-enYvelines, Montigny le Bretonneux, France ${ }^{5}$ Department of Medicine, Boston Children's Hospital, Boston, Massachusetts, USA ${ }^{6}$ Department of Pediatrics, Turku University Hospital, Turku, Finland

${ }^{7}$ Departments of Molecular Virology and Microbiology, and Pediatrics, Baylor College of Medicine, Houston, Texas, USA

\section{Correspondence to}

Dr Orianne Dumas, Inserm

UMRS 1168, VIMA, Aging and Chronic Diseases.

Epidemiological and Public Health Approaches, 16 Avenue Paul Vaillant Couturier Villejuif Cedex 94807, France; orianne.dumas@inserm.fr

Received 25 February 2016 Revised 4 May 2016 Accepted 28 May 2016 Published Online First 23 June 2016

\section{SLinked}

- http://dx.doi.org/10.1136/ thoraxjnl-2016-209012

\section{CrossMark}

To cite: Dumas 0

Mansbach JM, Jartti T, et al.

Thorax 2016;71:712-718.

\section{ABSTRACT}

Objective Although bronchiolitis is generally

considered a single disease, recent studies suggest heterogeneity. We aimed to identify severe bronchiolitis profiles using a clustering approach.

Methods We analysed data from two prospective, multicentre cohorts of children younger than 2 years hospitalised with bronchiolitis, one in the USA (20072010 winter seasons, $n=2207$ ) and one in Finland (2008-2010 winter seasons, $n=408$ ). Severe bronchiolitis profiles were determined by latent class analysis, classifying children based on clinical factors and viral aetiology.

Results In the US study, four profiles were identified. Profile A (12\%) was characterised by history of wheezing and eczema, wheezing at the emergency department (ED) presentation and rhinovirus infection. Profile $B$ $(36 \%)$ included children with wheezing at the ED presentation, but, in contrast to profile $A$, most did not have history of wheezing or eczema; this profile had the largest probability of respiratory syncytial virus infection. Profile C (34\%) was the most severely ill group, with longer hospital stay and moderate-to-severe retractions. Profile D (17\%) had the least severe illness, including non-wheezing children with shorter length of stay. Two of these profiles ( $A$ and $D$ ) were replicated in the Finnish cohort; a third group ('BC') included Finnish children with characteristics of profiles $B$ and/or $C$ in the US population.

Conclusions Several distinct clinical profiles (phenotypes) were identified by a clustering approach in two multicentre studies of children hospitalised for bronchiolitis. The observed heterogeneity has important implications for future research on the aetiology, management and long-term outcomes of bronchiolitis, such as future risk of childhood asthma.

\section{INTRODUCTION}

Bronchiolitis is one of the most common infections in early childhood, ${ }^{1}$ and remains a clinical diagnosis. ${ }^{2}$ Although bronchiolitis is generally considered a single disease entity, the clinical course and severity are highly variable, ${ }^{13} 4$ and recent studies have suggested that bronchiolitis is heterogeneous. ${ }^{3} 56$ For instance, most children with bronchiolitis have a mild-to-moderate disease course, but approximately 3\% require hospitalisation (severe bronchiolitis). ${ }^{1}$ Even among hospitalised children, the two most common aetiological viruses, respiratory syncytial virus (RSV) and rhinovirus, are associated with different short-term clinical outcomes

\section{Key messages}

What is the key question?

- Can several distinct clinical profiles be identified among children with severe bronchiolitis?

\section{What is the bottom line?}

- Four distinct clinical profiles were identified by latent class analysis in a US multicentre cohort of children hospitalised with bronchiolitis, and consistent results were observed in a parallel, but separate, Finnish cohort.

\section{Why read on?}

- The clinical diagnosis of bronchiolitis is likely to include separate entities; this heterogeneity has important implications for research on the aetiology, management and long-term outcomes of bronchiolitis, such as future risk of childhood asthma.

(eg, hospital length of stay, risk of relapse). ${ }^{4}{ }^{7-9}$ In addition, although most hospitalised children have a monophasic illness pattern, they have a wide range of time to recovery. ${ }^{10}$ Importantly, beyond these short-term differences, heterogeneity has also been observed in long-term outcomes, such as the risk of developing recurrent wheezing and asthma. ${ }^{11-13}$ Studies have suggested that more severe bronchiolitis and rhinovirus infections in early childhood are strongly associated with an increased risk of subsequent recurrent wheezing and asthma. ${ }^{11}$ 14-17 However, the exact role of early infections in the pathogenesis of asthma remains unclear. ${ }^{11-13}$

Studies examining subgroups of patients with bronchiolitis generally classify children according to a limited number of characteristics (eg, RSV status, wheezing) or to a single dimension (eg, severity score). Compared with this simple approach, the benefits of multidimensional approaches in addressing a heterogeneous disease (with regard to its aetiology, pathobiology or treatments) have been demonstrated for other respiratory/allergic disorders, such as asthma, wheezing illness of childhood or atopy. ${ }^{18-21}$ Bronchiolitis heterogeneity could reflect the existence of several underlying phenotypes, or clinically meaningful profiles, that have different roles in the development of childhood 
asthma. $^{3} 5$ However, to date, no consensus has emerged regarding the definition of subgroups of children with bronchiolitis who may respond differently to medications and/or have different short-term or long-term outcomes. ${ }^{22-26}$

Clustering statistical methods are useful to identify profiles that summarise shared aspects of a disease within different groups of affected patients. Latent class analysis (LCA) is a 'hypothesis-free' approach, which is used to identify more homogeneous subgroups of patients from a large set of observed clinical characteristics. $^{18} 2027$ This method has proven useful, for instance, to identify wheezing or respiratory/allergic phenotypes in children or adults. ${ }^{18} 202127$ To date, the technique has never been applied to children with bronchiolitis.

The aim of the present study was to identify and describe discrete profiles of children with severe bronchiolitis. For this purpose, we first applied LCA in a prospective, multicentre study of more than 2200 children hospitalised with bronchiolitis in the USA. Then, the LCA was replicated in a parallel, but separate, multicentre study of 408 children hospitalised with bronchiolitis in Finland. We also investigated potential differences in the treatments given to children with the different profiles of severe bronchiolitis.

\section{MATERIAL AND METHODS Study design}

We conducted a prospective, multicentre cohort study of US children hospitalised with bronchiolitis during the 2007-2010 winter seasons (November-March). ${ }^{4}$ The study was part of the Multicenter Airway Research Collaboration (MARC-30 USA), a programme of the Emergency Medicine Network (EMNet, http://www.emnet-usa.org). Investigators at 16 sites across 12 US states used a standardised protocol to enrol 2207 children who were diagnosed by the attending physician as having bronchiolitis (as defined by the American Academy of Pediatrics: ${ }^{28}$ acute respiratory illness with some combination of rhinitis, cough, tachypnoea, wheezing, crackles and retractions), and were younger than 2 years. Children were enrolled from the inpatient unit (ward) and the intensive care unit (ICU). The Finnish counterpart study (MARC-30 Finland) ${ }^{8}$ used an identical protocol to enrol 408 children (2008-2010 winter seasons) from three hospitals.

All patients were treated at the discretion of the treating physician. Although RSV status may have been known for some subjects during the hospitalisation from local testing, viral aetiology was generally not known by the treating physician. Written informed consent was obtained from a parent or guardian of all patients included in the study. The institutional review board at each participating hospital approved the study.

\section{Data collection}

The investigators conducted a structured interview that assessed patients' demographic characteristics, medical and environmental history and details of the acute illness. ${ }^{4}$ Emergency department (ED) and daily hospital medical records provided further clinical data; data extracted from records onsite were manually reviewed at the EMNet coordinating centre. Researchers collected nasopharyngeal aspirates using a standardised protocol, and performed testing for a large panel of respiratory pathogens (see online supplementary material) using real-time PCR assays at the core laboratory (Houston, Texas, USA). ${ }^{4}$

\section{Statistical analyses}

Severe bronchiolitis profiles were determined by LCA. The children were classified based on their medical history (history of wheezing or eczema and family history of asthma, based on parents' interview), the bronchiolitis clinical characteristics (main features typically monitored in clinical practice, including data from ED presentation to inpatient discharge, based on hospital medical records) and the viral aetiology from PCR, with a focus on the most commonly detected viruses: RSV and rhinovirus. We first selected a large set of 18 variables (see online supplementary material). This set of variables was reduced using a multiple correspondence analysis (MCA) to select the most relevant variables for cluster analysis (see online supplementary table S1). ${ }^{27}{ }^{29}$ LCA was then performed based on the selected variables. The number of classes was determined by comparing models with two to six classes using the Bayesian information criteria (see online supplementary figure S1).

Analyses were conducted separately in the US and Finnish studies, and the profiles derived in each study were compared. Because the clinical definition of bronchiolitis sometimes places an upper age limit of 12 months, ${ }^{5}{ }^{30}$ in the US study, analyses were conducted (a) in all children younger than 2 years $(\mathrm{n}=2207)$, and (b) among children younger than 12 months (infants, $\mathrm{n}=1816$, analyses presented in online supplementary tables S3 and S4). As no major differences in the resulting profiles were observed between both groups, analyses in the Finnish study included all children (younger than 2 years, $n=408$ ) to preserve sample size.

We then investigated the association between the severe bronchiolitis profiles and treatment with antibiotics and typical asthma medications (inhaled bronchodilators, systemic or inhaled corticosteroids) during the ED visit and inpatient stay. We used logistic regression to adjust for age, sex and race/ ethnicity.

\section{RESULTS}

The mean age of the US children was 5.8 months (SD: 5.2 ), and $41 \%$ were female. Hospital length of stay was $\geq 3$ days for $44 \%$ of the children, and $18 \%$ were admitted to the ICU. The mean age of the Finnish children was 8.1 months (SD: 6.7) and 39\% were female. Compared with US children, hospital length of stay in Finnish children was shorter ( $\geq 3$ days: $40 \%$ ), and only $4 \%$ were admitted to the ICU. The US and Finnish children also differed with respect to viral aetiology: $72 \%$ (USA) versus $43 \%$ (Finnish) were infected with RSV, 26\% versus 32\% with rhinovirus, $13 \%$ versus $3 \%$ were infected with both RSV and rhinovirus and $15 \%$ versus $28 \%$ had non-RSV and non-rhinovirus infection. Finally, Finnish children more often had a history of wheezing (Finnish: $37 \%$ vs USA: $23 \%$ ) and eczema (29\% vs 14\%). More detailed sociodemographic and clinical characteristics of the cohorts are presented in tables 1, 2 (USA) and 3, 4 (Finnish).

\section{LCA in MARC-30 USA}

Among the 18 characteristics selected to classify the children, 9 variables were included in the LCA. A four-class model was retained as the best-fitting model. The profiles, labelled with letters $\mathrm{A}$ to $\mathrm{D}$, are presented in table 1 :

- Profile A children (12\%) were characterised by history of wheezing (probability: 48\%), history of eczema (32\%) and wheezing at the ED presentation (90\%). Profile A also differed from other profiles by the high probability of rhinovirus infection $(66 \%$ in profile A vs $12 \%-24 \%$ in other profiles) and lowest probability RSV infection $(18 \%$ in profile A vs 63\%-89\%).

- Profile B (36\%) included children with wheezing at the ED presentation $(82 \%)$, but, in contrast to profile A, most did 
Table 1 Description of the children hospitalised for severe bronchiolitis according to the profiles (A to D) identified by LCA, for the clinical characteristics introduced in the LCA model, MARC-30 USA, $n=2207$

\begin{tabular}{|c|c|c|c|c|c|}
\hline & \multirow[b]{2}{*}{ All } & \multicolumn{4}{|l|}{ Profiles } \\
\hline & & A $(12 \%)$ & B (36\%) & C (34\%) & $D(17 \%)$ \\
\hline History of wheezing & 23 & 48 & 21 & 19 & 12 \\
\hline History of eczema & 14 & 32 & 15 & 10 & 7 \\
\hline Wheeze at ED presentation & 64 & 90 & 82 & 63 & 11 \\
\hline Cough at ED presentation & 86 & 92 & 93 & 83 & 74 \\
\hline \multicolumn{6}{|l|}{ Retractions at ED presentation } \\
\hline None & 24 & 18 & 19 & 11 & 61 \\
\hline Mild & 45 & 47 & 56 & 39 & 34 \\
\hline Moderate to severe & 31 & 35 & 24 & 50 & 5 \\
\hline \multicolumn{6}{|l|}{ Hospital length of stay (days) } \\
\hline$<3$ & 56 & 82 & 75 & 16 & 77 \\
\hline $3-6$ & 33 & 15 & 24 & 58 & 18 \\
\hline$\geq 7$ & 11 & 3 & $<1$ & 27 & 5 \\
\hline \multicolumn{6}{|c|}{ Retractions during hospital stay } \\
\hline None & 34 & 44 & 47 & 2 & 69 \\
\hline Mild & 46 & 47 & 53 & 46 & 30 \\
\hline Moderate to severe & 20 & 9 & $<1$ & 52 & $<1$ \\
\hline \multicolumn{6}{|l|}{ Viral aetiology } \\
\hline RSV & 72 & 18 & 89 & 82 & 63 \\
\hline Rhinovirus & 26 & 66 & 12 & 24 & 23 \\
\hline \multicolumn{6}{|c|}{$\begin{array}{l}\text { Results are expressed as \% (observed proportion in the study population ('All'), and } \\
\text { probability of individuals presenting the characteristics within profiles A to D). } \\
\text { Profile A: history of wheezing/eczema, wheezing at ED presentation, more often } \\
\text { rhinovirus. Profile B: wheezing at ED presentation, more often RSV only. Profile C: } \\
\text { most severe. Profile D: non-wheezing at ED presentation, least severe. } \\
\text { ED, emergency department; LCA, latent class analysis; RSV, respiratory syncytial virus. }\end{array}$} \\
\hline
\end{tabular}

not have history of wheezing or eczema. This profile had the largest probability of RSV infection (89\%).

- Profile C (34\%) was the most severely ill group: children were more likely to have moderate-to-severe retractions (50\% at presentation and 52\% during hospital stay in profile C vs 5\%-35\% and 1\%-9\% in other profiles), and to be hospitalised $\geq 7$ days ( $27 \%$ vs $1 \%-5 \%$ in other profiles). Profile $\mathrm{C}$ also had a large probability of RSV infection (82\%).

- Profile D (17\%) was the least severely ill group, and included mostly non-wheezing children ( $11 \%$ at ED presentation) with no or mild retractions, and a shorter hospital length of stay.

Table 2 shows the distribution across the four profiles of sociodemographic and clinical characteristics not used in the LCA classification. The profiles largely differed in age: profile A included older children ( $71 \%$ older than 6 months), while the youngest children were more often in the most severe profile (profile C, 37\% younger than 2 months) or in the least severe non-wheezing profile (profile D, $45 \%$ younger than 2 months). Significant associations were observed between profiles and both sex and race, with notably a higher proportion of boys and black children in profile A. A suggestive association was observed with parental history of asthma $(p=0.08)$, which was more common in profile A. As expected, fever and inadequate oral intake at ED presentation, ICU admission and mechanical ventilation were observed more often in the most severe group (profile C).

Associations between the bronchiolitis profiles and treatment during the ED visit and inpatient stay are shown in figure 1.
Compared with profile B (reference group), profile A children were more often treated with asthma medication (bronchodilator during the inpatient stay and corticosteroids, both in the ED and during the inpatient stay). Profile $\mathrm{C}$ children were more often treated with all three medications during the inpatient stay. Profile D children were more often treated with antibiotics. Analysis of the number of treatments received during the inpatient stay showed that profile $\mathrm{A}$ and profile $\mathrm{C}$ children more often had repeated treatment with asthma medications: respectively $49 \%$ and $54 \%$ of them received two or more inhaled bronchodilator treatments, while $30 \%$ among profile B children. Similarly, 22\% (profile A) and 22\% (profile C) were treated with corticosteroids for at least 2 days, while 10\% among profile B children.

\section{Replication of the LCA in MARC-30 Finland}

The variable selection by MCA was conducted as closely as possible to the MARC-30 US analysis, although the variables selected slightly differed because of the lower prevalence of cases with ICU admission or hospitalised $\geq 7$ days in the Finnish cohort, and to avoid selecting variables with a higher rate of missing values (see online supplementary material). Among a first set of 13 characteristics selected to classify the children, 9 variables were included in the LCA. A three-class model was retained as the best-fitting model. To match with MARC-30 US labels, MARC-30 Finland profiles were labelled 'A', 'BC' and 'D', and are presented in table 3:

- Profile A was the most prevalent in the Finnish cohort (47\%). Similarly to US results, profile A regrouped children history of wheezing (56\%), history of eczema (40\%) and wheezing at the ED presentation (93\%), and infected with rhinovirus (54\%).

- Profile BC (38\%) was characterised by RSV infection (98\%), and an intermediate probability of wheezing at the ED presentation (62\%), and a low probability of children with history of wheezing or eczema. While moderate-to-severe retractions at $\mathrm{ED}$ presentation were more frequent in profile A $(65 \%)$, profile BC children were more likely to have other indicators of severe illness (inadequate oral intake: 39\%, length of stay $\geq 3$ days: $46 \%$ ).

- Profile D (15\%) was the least severely ill group, and included mostly children without wheezing (22\% at ED presentation), without cough (20\%), and with no or mild retractions.

As in the US study, profile A included older children and a higher proportion of boys than other profiles (table 4). However, in contrast to the US results, profiles A and BC had similar proportions of children with parental history of asthma. Associations between the severe bronchiolitis profiles and treatment are shown in online supplementary figure S2. Treatment patterns differed according to profiles, with again higher treatment with asthma medication in the Finnish children with profile A.

\section{DISCUSSION}

Several distinct clinical profiles of severe bronchiolitis were identified by a hypothesis-free statistical clustering approach in two multicentre studies of children hospitalised for bronchiolitis. Four profiles were identified in a large US sample; two of them (A and B) included mostly children with wheezing at ED presentation, but differed markedly in terms of medical history (history of wheezing and/or eczema more frequent in profile A), and viral aetiology (more rhinovirus infection in profile A vs more RSV infection in profile B). The LCA also clearly identified a group of children with a higher severity of illness (profile C), but who neither had distinctive wheezing patterns nor viral 
Table 2 Characteristics of the children hospitalised for severe bronchiolitis according to the profiles (A to D) identified by LCA, for additional sociodemographic and clinical characteristics not introduced in the LCA model, MARC-30 USA, $n=2207$

\begin{tabular}{|c|c|c|c|c|c|c|}
\hline & \multirow[b]{2}{*}{ All } & \multicolumn{4}{|l|}{ Profiles } & \multirow[b]{2}{*}{ p Value } \\
\hline & & $A(12 \%)$ & B $(36 \%)$ & C (34\%) & $D(17 \%)$ & \\
\hline \multicolumn{7}{|l|}{ Age (months) } \\
\hline$<2$ & 28 & 7 & 20 & 37 & 45 & $<0.001$ \\
\hline $2-5.9$ & 35 & 22 & 41 & 36 & 30 & \\
\hline $6-11.9$ & 22 & 41 & 24 & 18 & 15 & \\
\hline$\geq 12$ & 14 & 30 & 15 & 9 & 10 & \\
\hline Girls & 41 & 33 & 41 & 40 & 45 & 0.01 \\
\hline \multicolumn{7}{|l|}{ Race } \\
\hline White & 61 & 57 & 60 & 61 & 69 & $<0.001$ \\
\hline Black/African-American & 24 & 32 & 25 & 23 & 21 & \\
\hline Other & 14 & 11 & 15 & 16 & 10 & \\
\hline Hispanic ethnicity & 36 & 32 & 38 & 34 & 40 & 0.07 \\
\hline Parental history of asthma & 31 & 37 & 32 & 31 & 27 & 0.08 \\
\hline \multicolumn{7}{|l|}{ ED presentation } \\
\hline Fever & 29 & 23 & 30 & 32 & 27 & 0.05 \\
\hline Apnoea & 7 & 6 & 4 & 10 & 10 & $<0.001$ \\
\hline Inadequate oral intake & 50 & 38 & 46 & 60 & 45 & $<0.001$ \\
\hline \multicolumn{7}{|l|}{ ICU and intubation/CPAP } \\
\hline No ICU & 82 & 86 & 94 & 64 & 88 & $<0.001$ \\
\hline ICU without intubation/CPAP & 11 & 11 & 5 & 18 & 8 & \\
\hline ICU with intubation/CPAP & 7 & 3 & 1 & 17 & 4 & \\
\hline \multicolumn{7}{|l|}{ Viral aetiology } \\
\hline RSV only & 49 & 0 & 65 & 52 & 46 & $<0.001$ \\
\hline Rhinovirus only & 8 & 38 & 0 & 3 & 10 & \\
\hline RSV and rhinovirus & 13 & 8 & 10 & 20 & 9 & \\
\hline RSV and other non-rhinovirus & 10 & 0 & 15 & 10 & 6 & \\
\hline Rhinovirus and other non-RSV & 5 & 27 & 0 & 2 & 5 & \\
\hline Non-RSV and non-rhinovirus & 15 & 28 & 9 & 13 & 24 & \\
\hline \multicolumn{7}{|l|}{ Number of pathogens } \\
\hline No pathogen identified & 6 & 10 & 4 & 6 & 10 & $<0.001$ \\
\hline 1 & 64 & 53 & 71 & 61 & 65 & \\
\hline 2 & 24 & 29 & 22 & 27 & 20 & \\
\hline$\geq 3$ & 5 & 8 & 4 & 6 & 4 & \\
\hline
\end{tabular}

aetiologies. Children in profile D had the least severe illness, and no wheezing symptoms. Two of these profiles (A and D) were replicated in a Finnish cohort of children hospitalised for bronchiolitis; a third group ('BC') included children with characteristics of profiles $\mathrm{B}$ and/or $\mathrm{C}$ that we identified in the US population.

The positive association between early childhood rhinovirus-induced respiratory infections and risk of childhood asthma is well known. ${ }^{11} 12$ However, whether this association is causal or reflects an underlying susceptibility to both early infection and asthma remains unclear. ${ }^{11}{ }^{13}{ }^{31}$ It has been suggested that severe rhinovirus-induced infections may be a susceptibility marker for development of childhood asthma in predisposed children, rather than an inducer (causing asthma). ${ }^{11}$ 32-34 In the current analysis, a distinct bronchiolitis profile (A), characterised by prior history of wheezing and/or eczema, wheezing at ED presentation and more frequent rhinovirus infection, was identified independently in the US and Finnish cohorts. Profile A children were also more often boys, older and, in the US study, they had more often a parental history of asthma. Altogether, these results support the existence of a subgroup of children presenting early signs of asthma during a severe bronchiolitis episode.

Furthermore, profile A children were more often treated with bronchodilator and corticosteroids than other profiles (with the exception of US profile C, regrouping the most severely ill children). The children's medical history (especially wheeze at ED presentation and history of wheezing) probably influenced the physician's choice to initiate bronchodilator or corticosteroid therapy. However, it is interesting to note that most profile A children were treated with bronchodilators and corticosteroids repeatedly during the bronchiolitis episode. Our data do not allow further evaluation of response to treatment in the different profiles, which was not the purpose of this study. However, this question merits further research as current guidelines for the acute management of bronchiolitis do not differentiate disease subtypes. ${ }^{2}$ Bronchodilator and corticosteroid therapies are generally considered ineffective in bronchiolitis management, but the possibility that they may be beneficial in specific subgroups of patients has been suggested by several investigators worldwide. 2223253536 


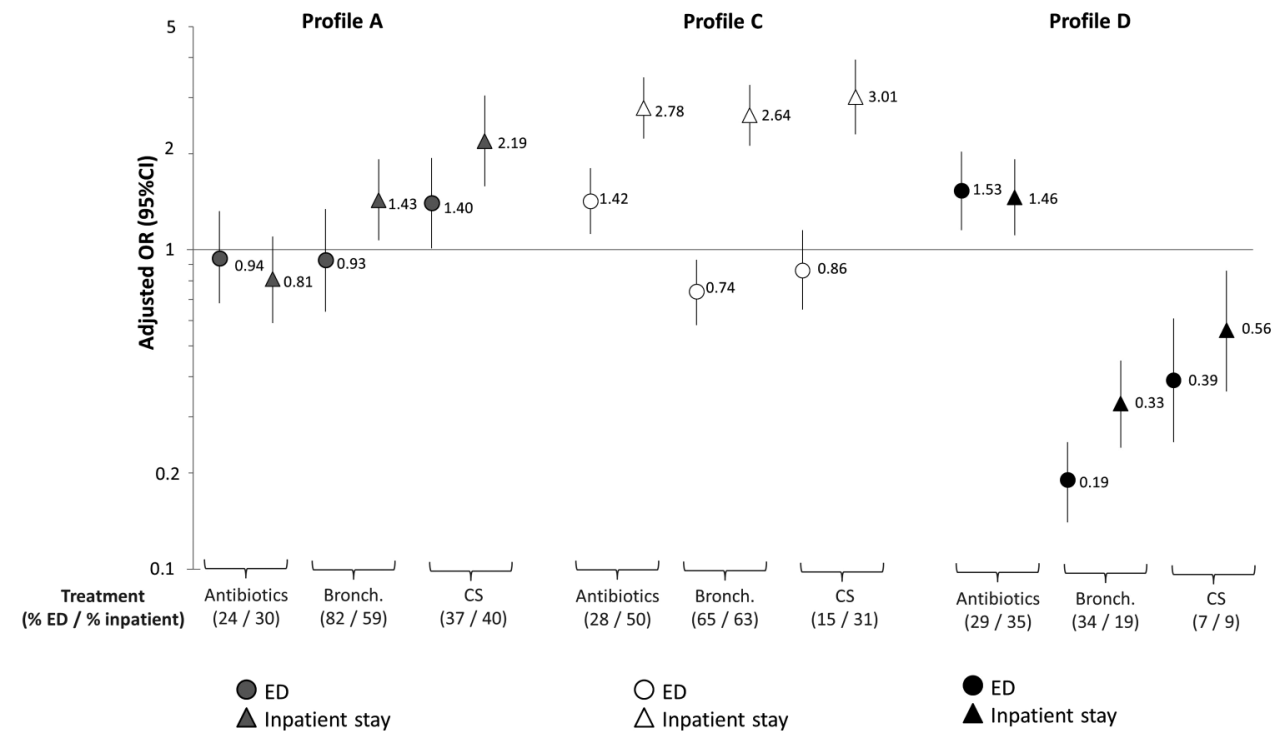

Figure 1 Associations between profiles (A to D) identified by latent class analysis (LCA) and treatment, in MARC-30 USA ( $n=2207$ ). Treatments: antibiotics, inhaled bronchodilators (bronch.), and systemic or inhaled corticosteroids (CS)) at emergency department (ED) presentation and during the inpatient stay. Results are presented as percentage of patients receiving treatment at ED presentation (\% ED) and during the inpatient stay (\%inpatient), and OR and $95 \% \mathrm{Cls}$, adjusted for age, sex and race/ethnicity. Profile B was used as the reference category (\% ED/\% inpatient for antibiotics: 23/30; bronchodilators: 76/44; corticosteroids: 21/17). Profile A: history of wheezing/eczema, wheezing at ED presentation, more often rhinovirus. Profile B: wheezing at ED presentation, more often respiratory syncytial virus (RSV) only. Profile C: most severe. Profile D: non-wheezing at ED presentation, least severe.

Bronchiolitis severity is another characteristic that has been independently associated with higher risk for development of childhood asthma. ${ }^{16}{ }^{17}$ The severity of bronchiolitis may also be a marker of a predisposition to develop childhood asthma, or actually play an aetiological role in its development. ${ }^{12} \mathrm{~A}$ profile regrouping the least severe cases (profile D) was observed in both populations. On the other hand, a profile regrouping the

Table 3 Description of the children hospitalised for severe bronchiolitis according to the profiles $(A, B C$ and $D)$ identified by LCA, for the clinical characteristics introduced in the LCA model, MARC-30 Finland, $n=408$

\begin{tabular}{|c|c|c|c|c|}
\hline & \multirow[b]{2}{*}{ All } & \multicolumn{3}{|l|}{ Profiles } \\
\hline & & $\begin{array}{l}A \\
(47 \%)\end{array}$ & $\begin{array}{l}\text { BC } \\
(38 \%)\end{array}$ & $\begin{array}{l}\text { D } \\
(15 \%)\end{array}$ \\
\hline History of wheezing & 37 & 56 & 15 & 33 \\
\hline History of eczema & 29 & 40 & 19 & 22 \\
\hline Wheeze at ED presentation & 69 & 93 & 62 & 22 \\
\hline Cough at ED presentation & 65 & 78 & 70 & 20 \\
\hline $\begin{array}{l}\text { Inadequate oral intake at ED } \\
\text { presentation }\end{array}$ & 21 & 10 & 39 & 12 \\
\hline \multicolumn{5}{|l|}{ Retractions at ED presentation } \\
\hline None & 16 & 5 & 13 & 50 \\
\hline Mild & 41 & 30 & 55 & 40 \\
\hline Moderate to severe & 43 & 65 & 31 & 10 \\
\hline Hospital length of stay $\geq 3$ days & 32 & 25 & 46 & 22 \\
\hline \multicolumn{5}{|l|}{ Viral aetiology } \\
\hline RSV & 43 & 8 & 98 & 21 \\
\hline Rhinovirus & 32 & 54 & $<1$ & 40 \\
\hline \multicolumn{5}{|c|}{$\begin{array}{l}\text { Results are expressed as \% (observed proportion in the study population ["All"], anc } \\
\text { probability of individuals presenting the characteristics within profiles A, BC and D). } \\
\text { Profile A: history of wheezing/eczema, wheezing at ED presentation, more often } \\
\text { rhinovirus. Profile BC: wheezing at ED presentation, more often RSV only, most } \\
\text { severe. Profile D: nonwheezing at ED presentation, least severe. } \\
\text { ED, emergency department; LCA, Latent Class Analysis; RSV, respiratory syncytial } \\
\text { virus. }\end{array}$} \\
\hline
\end{tabular}

most severely ill children was identified in the US cohort (profile C), but was not fully replicated in the Finnish cohort. Besides disease severity, profile C was close to profile B (eg, high probability of RSV infection). The absence of identification of a separate severe profile (C) in the Finnish cohort may be explained by the lower proportion of patients with very severe bronchiolitis, potential differences in treatment practices and the smaller sample size. Alternatively, these results may indicate that a severe disease, rather than constituting a separate discrete profile (ie, profile C), is the end of a severity 'continuum'.

Although profile A children were clearly distinguished from the other profiles by a higher proportion of rhinovirus infection, not all of them were infected with rhinovirus. Some children in profile A were infected with other non-RSV pathogens or had no identified pathogen, with slightly different proportions in the US and Finnish studies. Moreover, only about half of profile A children in each cohort had history of wheezing. Finally, although profile A children were older, all age groups were represented, and similar profiles were observed in infants $<1$ year and in children $<2$ years. These observations underline the benefit of multidimensional approaches, as compared with the use of one single characteristic (eg, rhinovirus infection, history of wheezing, age cut-off), to capture the disease heterogeneity.

A notable strength of the current study is that our results are based on two multicentre studies (one of which included more than 2000 patients), from two different countries. Although all profiles were not directly replicated in the Finnish study, the overlap between US and Finnish profiles is noteworthy. Similar profiles were found despite between-country differences in virus prevalence and age. Moreover, in both studies, the consistency of the results was supported by statistical indicators (high mean class membership probability in each class), as well as the profiles interpretability.

Although the LCA approach is typically qualified as 'hypothesis free', variables are selected in relation to a specific research question. We chose to focus on the aspects of bronchiolitis heterogeneity which may help investigators to elucidate the positive 
Table 4 Characteristics of the children hospitalised for severe bronchiolitis according to the profiles (A, BC and D) identified by LCA, for additional socio-demographic and clinical characteristics not introduced in the LCA model, MARC-30 Finland, $\mathrm{n}=408$

\begin{tabular}{|c|c|c|c|c|c|}
\hline & \multirow[b]{2}{*}{ All } & \multicolumn{3}{|l|}{ Profiles } & \multirow[b]{2}{*}{ p Value } \\
\hline & & $A(47 \%)$ & BC (38\%) & $D(15 \%)$ & \\
\hline \multicolumn{6}{|l|}{ Age (months) } \\
\hline$<2$ & 16 & 3 & 31 & 18 & $<0.001$ \\
\hline $2-5.9$ & 26 & 15 & 42 & 20 & \\
\hline $6-11.9$ & 23 & 30 & 14 & 25 & \\
\hline$\geq 12$ & 35 & 52 & 13 & 38 & \\
\hline Girls & 38 & 32 & 45 & 43 & 0.04 \\
\hline Parental history of asthma & 24 & 25 & 28 & 15 & 0.10 \\
\hline \multicolumn{6}{|l|}{ ED presentation } \\
\hline Fever & 29 & 29 & 28 & 28 & 0.97 \\
\hline Apnoea & 2 & 0.5 & 4 & 0 & 0.03 \\
\hline \multicolumn{6}{|l|}{ Viral aetiology } \\
\hline RSV only & 35 & 1 & 89 & 5 & $<0.001$ \\
\hline Rhinovirus only & 23 & 39 & 0 & 30 & \\
\hline RSV and rhinovirus & 2 & 3 & 0 & 7 & \\
\hline RSV and other non-rhinovirus & 5 & 1 & 11 & 3 & \\
\hline Rhinovirus and other non-RSV & 7 & 12 & 0 & 8 & \\
\hline Non-RSV and non-rhinovirus & 28 & 44 & 0 & 47 & \\
\hline \multicolumn{6}{|l|}{ Number of pathogens } \\
\hline No pathogen identified & 14 & 22 & 0 & 24 & $<0.001$ \\
\hline 1 & 70 & 60 & 89 & 56 & \\
\hline 2 & 14 & 15 & 10 & 20 & \\
\hline$\geq 3$ & 2 & 3 & 0.6 & 0 & \\
\hline
\end{tabular}

association between (severe) bronchiolitis and risk of childhood asthma. The 18 characteristics selected initially included many features typically monitored in clinical practice. However, more objective measurement such as allergy and inflammatory biomarkers were not available, which is a limitation of the current study. Although profiles based on these features may not strictly meet the definition of a phenotype, they are likely markers of an underlying specific clinical condition, potentially of different pathobiology. Clinical studies with biomarkers, genetic and other '-omics' data and longer term follow-up are needed to further investigate this novel approach, and potentially, to identify bronchiolitis endotypes and establish more targeted and personalised disease management. ${ }^{37}$

Another limitation is the identification of disease profiles among only those children hospitalised for bronchiolitis, that is, those at the most severe end of the disease spectrum. Although profile D children may be similar to milder (outpatient) bronchiolitis cases, it is likely that different profiles would be identified in a larger patient population. However, our analysis revealed important heterogeneity even within this subgroup of children with severe bronchiolitis, which constitutes a high-risk group for development of childhood asthma. ${ }^{16} 17$

In summary, our analysis of two well-characterised, multicentre cohorts revealed that the clinical diagnosis of bronchiolitis is likely to include separate entities. The severe bronchiolitis profiles identified using a hypothesis-free statistical clustering approach differed according to prior history of wheezing/ eczema, wheezing at presentation, levels of acute severity and viral aetiology. The findings suggest potential differences in response to acute treatment and in future risk of childhood asthma. This observed heterogeneity has important implications for future research on the aetiology, management and long-term outcomes of bronchiolitis.

Acknowledgements The authors thank the MARC-30 US and MARC-30 Finland investigators for their dedication to bronchiolitis research.

Contributors OD: study conception and hypothesis delineation, statistical programming and data analysis, data interpretation, primary manuscript preparation; JMM, PAP and TJ: conceptualisation and design of the multicentre cohort studies, participation in the acquisition of the data, data interpretation and critical revision of the manuscript; $\mathrm{KH}$ : data interpretation and critical revision of the manuscript; AFS: participation in the acquisition of the data, data interpretation and critical revision of the manuscript; CAC: conceptualisation and design of the multicentre cohort studies, study conception and hypothesis delineation, participation in the acquisition of the data, data interpretation and critical revision of the manuscript. All authors approved the final version of the manuscript.

Funding This work was supported by the National Institutes of Health (Al-67693 and Al-87881); the Academy of Finland (132595 and 114034); the Sigrid Juselius Foundation, Helsinki, Finland; and the Foundation for Pediatric Research, Helsinki, Finland.

Disclaimer The content of this article is solely the responsibility of the authors and does not necessarily represent the official views of the National Institute of Allergy and Infectious Diseases or the National Institutes of Health, or the other institutes that provided support.

Competing interests None declared.

Patient consent Obtained.

Ethics approval US study: Institutional Review Board at each of the 16 participating hospitals; Finnish study: Institutional Review Board of Turku University Hospital.

Provenance and peer review Not commissioned; externally peer reviewed.

\section{REFERENCES}

1 Carroll KN, Gebretsadik T, Griffin MR, et al. Increasing burden and risk factors for bronchiolitis-related medical visits in infants enrolled in a state health care insurance plan. Pediatrics 2008;122:58-64. 
2 Ralston SL, Lieberthal AS, Meissner HC, et al. Clinical practice guideline: the diagnosis, management, and prevention of bronchiolitis. Pediatrics 2014;134: e1474-502.

3 Vicencio AG. Susceptibility to bronchiolitis in infants. Curr Opin Pediatr 2010;22:302-6.

4 Hasegawa K, Mansbach JM, Teach SJ, et al. Multicenter study of viral etiology and relapse in hospitalized children with bronchiolitis. Pediatr Infect Dis J 2014:33:809-13.

5 Jartti T, Lehtinen P, Vuorinen T, et al. Bronchiolitis: age and previous wheezing episodes are linked to viral etiology and atopic characteristics. Pediatr Infect Dis J 2009:28:311-17.

6 Vinci $\mathrm{R}$, Bauchner $\mathrm{H}$. Bronchiolitis, deception in research, and clinical decision making. JAMA 2014;312:699-700.

7 Mansbach JM, Piedra PA, Teach SJ, et al. Prospective multicenter study of viral etiology and hospital length of stay in children with severe bronchiolitis. Arch Pediatr Adolesc Med 2012;166:700-6.

8 Jartti T, Aakula M, Mansbach JM, et al. Hospital length-of-stay is associated with rhinovirus etiology of bronchiolitis. Pediatr Infect Dis J 2014;33:829-34.

9 Skjerven HO, Megremis S, Papadopoulos NG, et al. Virus type and genomic load in acute bronchiolitis: severity and treatment response with inhaled adrenaline. $J$ Infect Dis 2016:213:915-21.

10 Mansbach JM, Clark S, Piedra PA, et al. Hospital course and discharge criteria for children hospitalized with bronchiolitis. J Hosp Med 2015;10:205-11.

11 Rossi GA, Colin AA. Infantile respiratory syncytial virus and human rhinovirus infections: respective role in inception and persistence of wheezing. Eur Respir J 2015:45:774-89.

12 Feldman AS, He Y, Moore ML, et al. Toward primary prevention of asthma. Reviewing the evidence for early-life respiratory viral infections as modifiable risk factors to prevent childhood asthma. Am J Respir Crit Care Med 2015; 191:34-44.

13 Fuchs 0 , von Mutius E. Prenatal and childhood infections: Implications for the development and treatment of childhood asthma. Lancet Respir Med 2013;1:743-52.

14 Jackson DJ, Gangnon RE, Evans MD, et al. Wheezing rhinovirus illnesses in early life predict asthma development in high-risk children. Am J Respir Crit Care Med 2008;178:667-72.

15 Thomsen SF, van der Sluis S, Stensballe LG, et al. Exploring the association between severe respiratory syncytial virus infection and asthma: a registry-based twin study. Am J Respir Crit Care Med 2009;179:1091-7.

16 Bacharier LB, Cohen $R$, Schweiger $T$, et al. Determinants of asthma after severe respiratory syncytial virus bronchiolitis. J Allergy Clin Immunol 2012;130:91-100.

17 Carroll KN, Wu P, Gebretsadik T, et al. The severity-dependent relationship of infant bronchiolitis on the risk and morbidity of early childhood asthma. J Allergy Clin Immunol 2009;123:1055-61.

18 Siroux V, Garcia-Aymerich J. The investigation of asthma phenotypes. Curr Opin Allergy Clin Immunol 2011;11:393-9.

19 Vanfleteren LEGW, Kocks JWH, Stone IS, et al. Moving from the Oslerian paradigm to the post-genomic era: are asthma and COPD outdated terms? Thorax 2014;69:72-9.
20 Depner M, Fuchs O, Genuneit J, et al. Clinical and epidemiologic phenotypes of childhood asthma. Am J Respir Crit Care Med 2014;189:129-38.

21 Henderson J, Granell R, Heron J, et al. Associations of wheezing phenotypes in the first 6 years of life with atopy, lung function and airway responsiveness in mid-childhood. Thorax 2008;63:974-80.

22 Schroeder AR, Mansbach JM. Recent evidence on the management of bronchiolitis. Curr Opin Pediatr 2014;26:328-33.

23 Jartti T, Nieminen R, Vuorinen T, et al. Short- and long-term efficacy of prednisolone for first acute rhinovirus-induced wheezing episode. J Allergy Clin Immunol 2015;135:691-8.

24 Zorc JJ. Inhaled epinephrine does not shorten hospital stay for infants with bronchiolitis destined to develop repeated bronchospasm. Lancet Respir Med 2015:3:665-7.

25 Mazur NI, Martinón-Torres F, Baraldi E, et al. Lower respiratory tract infection caused by respiratory syncytial virus: current management and new therapeutics. Lancet Respir Med 2015;3:888-900.

26 Skjerven HO, Rolfsjord LB, Berents TL, et al. Allergic diseases and the effect of inhaled epinephrine in children with acute bronchiolitis: Follow-up from the randomised, controlled, double-blind, Bronchiolitis ALL trial. Lancet Respir Med 2015;3:702-8

27 Herr M, Just J, Nikasinovic L, et al. Risk factors and characteristics of respiratory and allergic phenotypes in early childhood. J Allergy Clin Immunol 2012;130:389-96.

28 American Academy of Pediatrics Subcommittee on Diagnosis and Management of Bronchiolitis. Diagnosis and management of bronchiolitis. Pediatrics 2006:118:1774-93.

29 Siroux V, Basagaña X, Boudier A, et al. Identifying adult asthma phenotypes using a clustering approach. Eur Respir J 2011;38:310-17.

30 Hyvärinen M, Ruotsalainen M, Korppi M. Outcome after bronchiolitis depends on disease definition. Thorax 2011;66:266-7; author reply 267

31 Mattes J, Murphy VE, Powell $H$, et al. Prenatal origins of bronchiolitis: protective effect of optimised asthma management during pregnancy. Thorax 2014:69:383-4.

32 Carroll KN, Gebretsadik T, Minton P, et al. Influence of maternal asthma on the cause and severity of infant acute respiratory tract infections. J Allergy Clin Immunol 2012;129:1236-42.

33 Jackson DJ, Evans MD, Gangnon RE, et al. Evidence for a causal relationship between allergic sensitization and rhinovirus wheezing in early life. Am J Respir Crit Care Med 2012;185:281-5.

34 Calışkan M, Bochkov YA, Kreiner-Møller E, et al. Rhinovirus wheezing illness and genetic risk of childhood-onset asthma. N Engl J Med 2013;368:1398-407.

35 Alansari K, Sakran M, Davidson BL, et al. Oral dexamethasone for bronchiolitis: a randomized trial. Pediatrics 2013;132:e810-16.

36 DeLaMora P, Moscona A. Bronchiolitis: too-familiar yet too-mysterious disease of childhood. Arch Pediatr Adolesc Med 2012;166:769-70.

37 Belgrave D, Simpson A, Custovic A. Challenges in interpreting wheeze phenotypes: the clinical implications of statistical learning techniques. Am J Respir Crit Care Med 2014;189:121-2. 